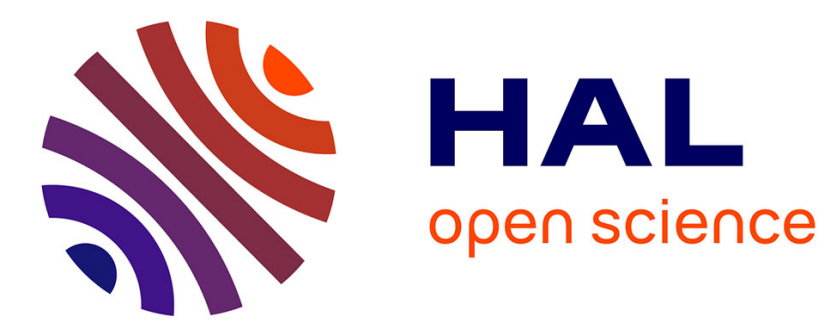

\title{
Dynamics of ultrafast stress generation in metals
}

\author{
O. Wright
}

\section{To cite this version:}

O. Wright. Dynamics of ultrafast stress generation in metals. Journal de Physique IV Proceedings, 1994, 04 (C7), pp.C7-701-C7-704. 10.1051/jp4:19947164 . jpa-00253224

\section{HAL Id: jpa-00253224 https://hal.science/jpa-00253224}

Submitted on 1 Jan 1994

HAL is a multi-disciplinary open access archive for the deposit and dissemination of scientific research documents, whether they are published or not. The documents may come from teaching and research institutions in France or abroad, or from public or private research centers.
L'archive ouverte pluridisciplinaire HAL, est destinée au dépôt et à la diffusion de documents scientifiques de niveau recherche, publiés ou non, émanant des établissements d'enseignement et de recherche français ou étrangers, des laboratoires publics ou privés. 


\title{
Dynamics of ultrafast stress generation in metals
}

O.B. Wright(1) Electronics Research Laboratories, Nippon Steel Corporation, 5-10-1 Fuchinobe, Sagamihara,
Kanagawa 229, Japan

\begin{abstract}
Using a time-resolved optical pump and probe scheme with laser beam deflection detection, the dynamics of ultrafast stress generation in copper by picosecond optical pulses has been investigated. The phonon strain pulse shape can be explained through the nonequilibrium coupling of the electrons and the lattice.
\end{abstract}

When an ultrashort optical pulse is incident on a metal, electrons near the surface are excited to higher available states. The propagation, diffusion and relaxation of the hot electrons determine the final profile of the stress pulse travelling away from the metal surface, and result in a broadening of this pulse.[1,2] Preliminary results supporting this reasoning were recently obtained for group-VI transition metals using a beam deflection technique for direct stress pulse detection from ultrafast surface vibrations.[3, 4] Here I describe similar results for copper and show how the stress pulse shapes can be understood by considering the nonequilibrium coupling between the electrons and the lattice.

Pump pulses of 630-nm wavelength, energy $\sim 1 \mathrm{~nJ}$ and of duration $3 \mathrm{ps}$ (FWHM) are used to excite the stress pulses. Changes in surface profile associated with stress pulses returning to the surface lead to an angular deflection $(\delta \theta)$ of a delayed probe beam, proportional to the surface displacement, which is monitored with a dual-cell photodiode.

Figure 1 shows the results for $\delta \theta$ as a function of delay time for a sputtered polycrystalline (grain size $\sim 20-200 \mathrm{~nm}$ ) Cu film of thickness $450 \mathrm{~nm}$ on a silica substrate. Echoes arise from stress pulses multiply reflected inside the film. The approach commonly used to interpret the echo shape is a one-dimensional thermoelastic model in the acoustic near field:[2] energy absorbed in the region of the optical skin depth ( $\zeta=16 \mathrm{~nm}$ ) results in a thermal expansion that launches a longitudinal stress pulse $\sigma_{33}(z-v t)$ [ $v$ the sound velocity] into the metal, as shown in Fig. 2(a). Integrating with respect to depth $z$ gives the normal surface displacement $u_{33}(t)$, shown in curve (a) of Fig. 1 . (The surface velocity $\mathrm{d} u_{33} / \mathrm{d} t$ is proportional to $\sigma_{33}(t),[3]$ allowing the stress pulse shape to be measured directly.) The predicted pulse duration ( $\sim 10 \mathrm{ps})$, approximately twice the acoustic transit time $(2 / / v)$ across

(1) Present address: Consiglio Nazionale delle Ricerche (CNR), Istituto di Acustica "O.M. Corbino", Via Cassia 1216, 00189 Roma, Italy 


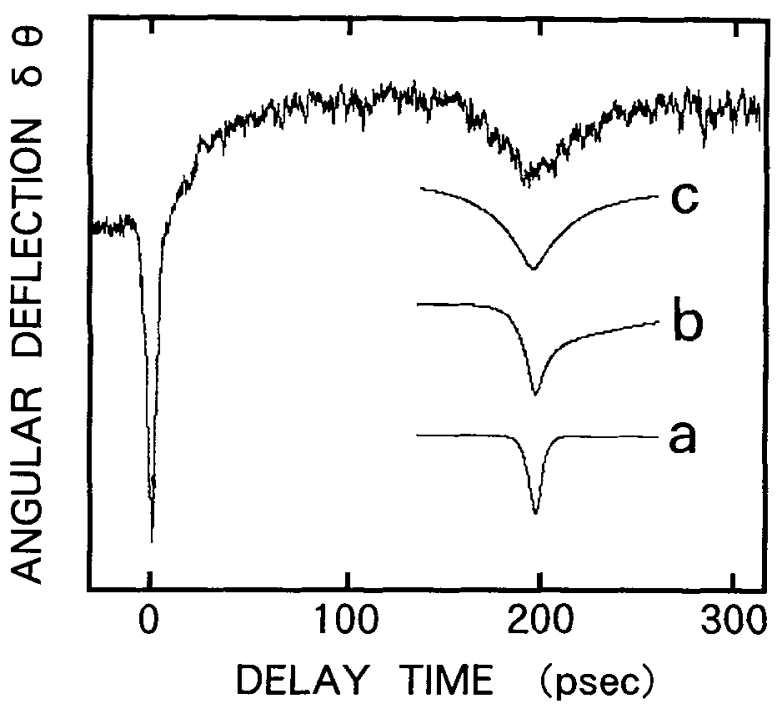

Fig. 1: Angular deflection of the probe laser beam against pump-probe delay time for a 450-nm film of Cu: (a) fit to first echo from the thermoelastic model; (b) fit from the thermoelastic model including thermal diffusion; (c) fit from the nonequilibrium model.

the skin depth, is much shorter than that ( $\sim 40 \mathrm{ps)} \mathrm{observed.} \mathrm{(The} \mathrm{effect} \mathrm{of} \mathrm{the} \mathrm{optical} \mathrm{pulse}$ duration is included in the theory.) A contribution to the echoes from radial gradients of the dielectric constants[3] is estimated to be small ( $<20 \%$, using data obtained with timeresolved reflectance detection).

Figure 2(b) and the curve (b) of Fig. 1 show refinements of the predictions to account for the effect of (Fourier) thermal diffusion on the stress pulse shapes. These curves were obtained by generalizing the expressions derived by Thomsen et al.[2] to a finite optical pulse duration:

$$
\sigma_{33}(z, t)=-\left.\frac{r C}{2} \int_{-\infty}^{\infty} \operatorname{sgn}\left[z-v\left(t-t^{\prime}\right)\right] \frac{\partial T}{\partial t}\right|_{\left(\left|z-v\left(t-t^{\prime}\right)\right|, t^{\prime}\right)} \mathrm{d} t^{\prime},
$$

where $p$ is the density, $\gamma$ the Gruneisen parameter, $C$ the specific heat capacity (per unit volume), $T$ is the temperature, and the point $t^{\prime}=0$ is excluded from the integral. A dimensionless parameter $D / v \zeta(\approx 1.6$ for $\mathrm{Cu}$, where $D$ is the thermal diffusivity) determines the pulse shapes. The derived pulse is broadened by a factor of about 2 compared to the prediction of the thermoelastic model. However, the experimental pulse widths are still significantly greater than this, and the predicted asymmetric pulse shape is not observed.

The excitation and relaxation of the electrons is a nonequilibrium process which can be expected to affect the stress generation. Stress broadening occurs because the electrons are able to diffuse (at supersonic velocities) a significant distance (compared to $\zeta$ ) before they 


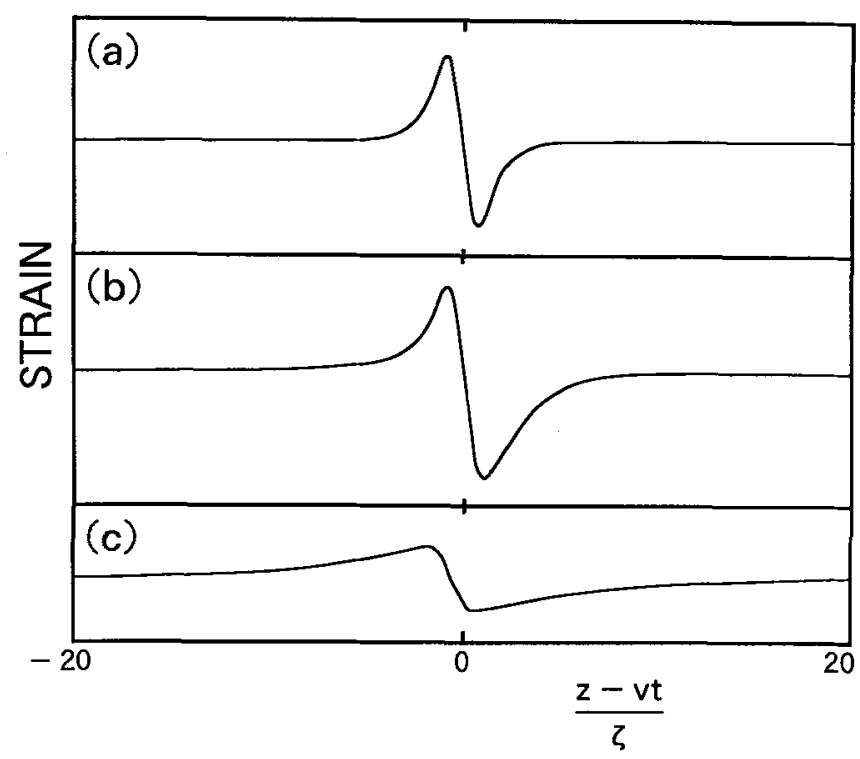

Fig. 2: Stress pulse shapes for $\mathrm{Cu}$, plotted as a function of the dimensionless parameter $(z-v t) / \bar{\zeta}$, calculated according to (a) the thermoelastic model, (b) the thermoelastic model including thermal diffusion, and (c) the nonequilibrium model for a pump fluence of $0.6 \mathrm{mJcm}^{-2}$. The scales for (a)(c) are the same.

lose their energy. By assuming that the electron and phonon distributions remain in separate pseudoequilibrium states each defined by a temperature ( $T_{\mathrm{e}}$ and $T_{\mathrm{i}}$, respectively), it is possible to calculate the evolution of these temperatures by a system of two coupled nonlinear differential equations:[5]

$$
\begin{gathered}
C_{\mathrm{e}}\left(T_{\mathrm{e}}\right) \frac{\partial T_{\mathrm{e}}}{\partial t}=\nabla \cdot\left(\mathrm{k} \nabla T_{\mathrm{e}}\right)-g\left(T_{\mathrm{e}}-T_{\mathrm{i}}\right)+P(z, t), \\
C_{\mathrm{i}} \frac{\partial T_{\mathrm{i}}}{\partial t}=g\left(T_{\mathrm{e}}-T_{\mathrm{i}}\right),
\end{gathered}
$$

where $g$ is the electron-phonon coupling constant, $\mathrm{k}$ is the thermal conductivity, $C_{\mathrm{e}}$ and $C_{\mathrm{i}}$ are specific heat capacities (per unit volume), and $P(z, t)$ is the absorbed laser power density [decaying exponentially with depth as $\exp (-z / \zeta)$ ]. These equations were solved numerically for the electron and lattice temperatures with a Gaussian temporal profile for the optical pulses. Then, by relating the generated stress to the changing temperature distribution through Eq. (1), the stress pulse shape can be calculated [see Fig. 2(c)]. 'The high frequency contribution from the electron temperature change is ignored because ultrasonic attenuation is expected to damp it out in this case. The pulse shape obtained using the value, $g=48 \times 1015 \mathrm{~W} / \mathrm{m}^{3} . \mathrm{K}$, for the electron-phonon coupling constant determined from other 
measurements[6] is in good agreement with experiment, as shown by the curve (c) in Fig. 1. The absolute amplitude (and sign) of the predictions are also in agreement with the data. (The amplitude of the curve (c) of Fig. 1 has been adjusted for the best fit.) Additional broadening effects from acoustic attenuation (due to grain boundary scattering)[7] are estimated to be small compared to the nonequilibrium broadening in this case. Similar results were obtained for films of the other noble metals Au and Ag.[8] The symmetry of the predicted pulse shapes is caused by the predominance of the effect of the spatial separation of the elemental acoustic sources (of amplitude $\propto \partial T_{\mathrm{i}} / \partial t$ ) compared to the duration of these sources. (An arbitrary distribution of impulsive sources all acting at the same time will produce an antisymmetric stress pulse and a symmetric surface displacement echo because of the $\pi$ phase change for stress reflection at the free surface, ignoring attenuation, diffraction or dispersion effects.) In constrast, the effect of the duration of these sources is important for the case of Fourier diffusion, leading to an asymmetric pulse shape.

In conclusion, the dynamics of stress generation by picosecond optical pulses in a metal has been investigated using copper films. The nonequilibrium coupling between the electrons and the lattice can account for the observed stress pulse shapes.

[1] V.E. Gusev, Opt. Commun. 94 (1992) 76.

[2] C. Thomsen, H. T. Grahn, H. J. Maris, and J. Tauc, Phys. Rev. B 34 (1986) 4129.

[3] O. B. Wright and K. Kawashima, Phys. Rev. Lett. 69 (1992) 1668.

[4] O. B. Wright and K. Kawashima, Jpn.J. Appl. Phys. 32 (1993) 2452.

[5] S. I. Anisimov, B. L. Kapeliovich, and T. L. Perelman, Zh. Eksp. Teor. Fiz. 66 (1974) 776 [Sov. Phys. JETP 39 (1975) 375].

[6] S. D. Brorson, A. Kazeroonian, J. S. Moodera, D. W. Face, T. K. Cheng, E. P. Ippen. M. S. Dresselhaus, and G. Dresselhaus, Phys. Rev. Lett. 64 (1990) 2172.

[7] B. Lehr, H. Ulrich, and O. Weis, Z. Phys. B 48 (1982) 23.

[8] O.B. Wright, Phys. Rev. B (March 1994). 